\title{
Pudilan xiaoyan oral liquid (PDL) inhibit the replication of influenza A virus through regulating TLR3/MyD88 signaling pathway
}

\section{Zheng Zhihui}

Yangzhou University Medical college

\section{Yuqian Zhang}

Yangzhou University Medical college

Gang Tian

Jumpcan Pharmaceutical Co. Ltd

\section{Zehua Wang}

Yangzhou University Medical college

\section{Ronghua Wang}

Yangzhou University Medical college

\section{Hailin Wei}

Yangzhou University Medical college

\section{Wenlei Wang}

Yangzhou University Medical college

\section{Qin Su}

Yangzhou University Medical college

\section{Rui Min}

Yangzhou University Medical college

Xiaoquan Wang

Yangzhou University

Pinghu Zhang ( $\square$ zhangpinghu@yzu.edu.cn )

Yangzhou University https://orcid.org/0000-0002-7600-4740

\section{Research}

Keywords: Influenza A virus, Pudilan xiaoyan oral liquid, Anti-inflammation, TLR3/MyD88/IRF7 signaling pathway, Acute lung injury

Posted Date: January 5th, 2022

DOI: https://doi.org/10.21203/rs.3.rs-1205788/v1 
License: (c) (i) This work is licensed under a Creative Commons Attribution 4.0 International License. Read Full License 


\section{Abstract \\ Background}

Pudilan Xiaoyan Oral Liquid (PDL) as a famous Chinese patent medicine has been widely used for treating upper respiratory tract infection. However, the antiviral effect of PDL remain unclear. Here, the antiviral effect of in vitro and in vivo of PDL against influenza A virus were for the first time investigated.

\section{Methods}

The in vitro inhibitory effect of PDL on influenza A virus was investigated using MDCK cell model. The in vivo inhibitory effect on influenza virus pneumonia was evaluated with the ICR female mice (14-16 g) model infected by influenza A virus (A/FM/1/47, H1N1, mouse-adapted). Moreover, expression levels of inflammatory cytokines including TNF-a, IP10, IL-10, IL-1 $\beta$, IL- 6 and IFN- $\gamma$ in lung tissue were measured by qRT-PCR. The potential mechanism of PDL against acute lung injury caused by influenza $A$ virus was investigated by RT-PCR and Western blot.

\section{Results}

Our results indicated that in vitro PDL has a broad-spectrum inhibitory effect on different subtypes of influenza A viruses and in vivo PDL could dose-dependently prevent weight loss of mice, increase food intake and reduce mortality caused by influenza A H1N1 virus. Furthermore, PDL could markedly improve the acute lung injury caused by influenza $A$ virus and significantly reduce the mRNA levels of inflammatory factors such as TNF-a, IP10, IL-10, IL-1ß, IL-6, and IFN- - . Mechanistic research indicated that the protective effect of PDL on viral pneumonia might be achieved by inhibiting TLR3/MyD88/IRAK4/TRAF3 signaling pathway.

\section{Conclusion}

PDL not only showed a good inhibitory effect on influenza A virus in vitro, but also exhibited a significant protective effect against lethal influenza virus infection in vivo. These findings provide evidence for the clinical treatment of influenza A virus infection with PDL.

\section{Background}

Influenza (Flu) is a common human respiratory disease caused by influenza A virus, which could result in pandemic outbreaks worldwide [1-5]. In 1918, the severe seasonal flu epidemic (H1N1) caused more than 50 million deaths in worldwide [6, 7]. Due to the high mutation rate of influenza A virus and poor cross-protective effect of different subtype influenza vaccines, the protective effect of vaccine is very limited[8]. Furthermore, the newly emerging cross-species transmission of avian influenza viruses such as 
$\mathrm{H} 5 \mathrm{~N} 1$ and $\mathrm{H} 7 \mathrm{~N} 9$, which could cause high mortality in patients, poses great challenges to human health [9-12]. An emerging of researches has reported that the acute lung injury induced by excessive inflammatory response caused by influenza $A$ virus is the main cause of high mortality in patients with influenza A virus infection $[6,8]$. Therefore, modulating of the excessive inflammatory response may be an effective therapy for flu treatment.

Pudilan xiaoyan oral liquid (PDL) as a famous Chinese patent medicine, which is composed of Taraxacum mongolicum Hand.-Mazz., Scutellaria baicalensis Georgi, Corydalis bungeana Turcz., and Radix Isatidis seu Baphicacanthii, has been widely used to treat upper respiratory tract infection diseases, pharyngitis, tonsillitis in clinical [13-15]. A large number of clinical studies have indicated that antiinflammatory is the main mechanism of PDL for treating these above diseases [13]. However, whether PDL has anti-influenza virus activity remains unclear. In this study, the antiviral effect of PDL on influenza A virus in vitro and in vivo was for the first time investigated. Our results showed that PDL could directly inhibit the replication of seasonal influenza A virus (H1N1 and H3N2) and showed a good protective effect against cell pathogenic effect induced by influenza A viruses. Furthermore, PDL effectively increased the survival rate, prolonged the median survival time of mice, and reduced lung injury by suppressing the inflammatory cytokine expression via inhibition of the TLR3/MyD88/NF-KB signaling. These findings provide evidence for the clinical treatment of influenza A virus infection with PDL in the future.

\section{Methods}

\section{Reagents}

Modified Eagle's medium (MEM) (SH30022.01) and 0.25\% trypsin (SH40003.01) were obtained from Hyclone (Shanghai, China). Fetal bovine serum (1531294) was purchased from Biological Industries (Israel). HiScript II 1st Strand cDNA Synthesis Kit (R222) and AceQ Universal SYBR qPCR Master Mix (Q511) were purchased from Vazyme (Nanjing, China). Primary antibodies against TLR3 (AF0300), MyD88 (AF7524), TRAF3 (AF8220), TRAF6 (AF8223), IRF7 (AF2140) and ACTB (AA128) were purchased from Beyotime Institute of Biotechnology (Shanghai, China).

PDL was provided by Jumpcan Pharmaceutical Co., Ltd (Taizhou, China) and was diluted with MEM (2\% fetal bovine serum, $2 \mu \mathrm{g} / \mathrm{ml}$ TPCK-treated trypsin, $100 \mathrm{U} / \mathrm{ml}$ penicillin and streptomycin). To further verify the active components of PDL, the fingerprint of PDL was analyzed by high-performance liquid chromatography (HPLC). Oseltamivir phosphate (S2597) was purchased from Selleck Chemicals Co., Ltd (Shanghai, China) and dissolved in dimethyl sulfoxide (DMSO) to get $40 \mathrm{mg} / \mathrm{ml}$ mother solution. Ribavirin injection $(100 \mathrm{mg} / \mathrm{ml}, 19022581)$ was purchased from Jiangsu Lianshui Pharmaceutical Co., Ltd (Lianshui, China). Working concentrations of oseltamivir phosphate and ribavirin were obtained by dilution with MEM as previously described [8].

Virus and cells 
Madin-Darby canine kidney (MDCK) and A549 cell lines were purchased from Wuhan Collection Center of Wuhan University, and were cultured MEM or DME/F12 medium with $10 \%$ fetal bovine serum, $100 \mathrm{U} / \mathrm{ml}$ penicillin and streptomycin, respectively. Influenza A virus strains including H1N1 (A/WSN/1/33, A/PR/8/33, A/FM1/1/33), H3N2 (A/YZ/201/2010), H5N1 (A/Duck/Jiangsu/Sheyang/2004), H7N9 (A/Chicken/Jiangsu/CZJTC4/2013) and H9N2 (A/Chicken/Shangdong/SKD1/2011) were provided by Key Lab. of Livestock and Poultry Infectious Diseases of the Ministry of Agriculture of Yangzhou University. The $50 \%$ tissue culture infective dose $\left(\mathrm{TCID}_{50}\right)$ of the seeding virus were titrated in MDCK cells and the $50 \%$ lethal dose $\left(\mathrm{LD}_{50}\right)$ of the seeding virus to ICR mice were determined by the Reed-Muench method [16]. All experiments were performed in BSL-2 or BSL-3 biosafety laboratory at Yangzhou University.

MTT assay

The cytotoxicity of drugs in MDCK and A549 cells was determined by MTT assay. Briefly, MDCK cells or A549 cells $\left(2 \times 10^{4}\right.$ cells/well) were plated into 96 -well cell plates for $12 \mathrm{~h}$ and then were exposed to drugs for $72 \mathrm{~h}$. After treatment, each well was added with $20 \mu \mathrm{l}$ of MTT $(5 \mathrm{mg} / \mathrm{ml})$. After incubation for more 4 $\mathrm{h}$, the medium was removed and $150 \mu \mathrm{l}$ of DMSO was added into each well to dissolve formazan. The absorbance per well was measured at $570 \mathrm{~nm}$ with a microplate reader (BioTek, USA). The non-toxic concentration and $50 \%$ cytotoxic concentration $\left(\mathrm{CC}_{50}\right)$ of drugs was determined as previously reported [17].

Anti-influenza virus activity of PDL in vitro

The anti-influenza viral effect of PDL was determined as previously described [18]. Briefly, MDCK cells $\left(2 \times 10^{4}\right.$ cells/well) were plated into 96 -well plates for 12 , and then were inoculated with $10 \mathrm{TCID}_{50}$ viruses for $1 \mathrm{~h}$. After inoculation, the plates were exposed with various concentrations of drugs for $96 \mathrm{~h}$ at $35^{\circ} \mathrm{C}$ under $5 \% \mathrm{CO}_{2}$. After $96 \mathrm{~h}, 30 \mu \mathrm{l}$ of cell supernatant per well was taken to 96 -well V-type hemagglutination plate and $25 \mu \mathrm{l}$ of detected by HA assay according to WHO guidelines for hemagglutination test. Plates were fixed with $100 \mu \mathrm{l}$ of a $10 \%$ formaldehyde solution. After removing the solution, the plates were stained with a $0.1 \%(\mathrm{w} / \mathrm{v})$ crystal violet staining. The minimum dilution without obvious toxicity was taken as the maximum non-toxic concentration $\left(\mathrm{TC}_{0}\right)$ of drugs, and $50 \%$ cytotoxic concentration $\left(\mathrm{TC}_{50}\right)$ was calculated according to the Reed-Muench method [16].

Anti-influenza virus effects of PDL in vivo

ICR female mice (14-16 g) were purchased from Comparative Medical Center for Yangzhou University and were raised in IVC on a $12 \mathrm{~h} \mathrm{light/dark} \mathrm{cycle} \mathrm{and} \mathrm{maintained} \mathrm{at} 22 \pm 2^{\circ} \mathrm{C}$. All animal experiments were approved by the Ethics Committee of Yangzhou University (202010002) according to the Chinese Animal Protection Act and the National Research Council Criteria. To investigate the anti-influenza effect of PDL against $\mathrm{H} 1 \mathrm{~N} 1$ virus in vivo, all mice were anesthetized with ether inhalation, and were inoculated intranasally with $25 \mu \mathrm{l}$ of viral suspension containing 5 LD $_{50}$ of influenza virus (A/FM/1/47, H1N1, 
mouse-adapted) or normal saline. After infection, the mice were orally administered with PDL (8.0, 6.0, 4.0 $\mathrm{ml} / \mathrm{kg} /$ day), ribavirin ( $80 \mathrm{mg} / \mathrm{kg} /$ day), or saline daily for 7 days. All mice were observed daily for 15 days. The weight loss, food intake, and mortality in each group were recorded daily. The protective effects of PDL against influenza A virus were evaluated by the survival time and mortality.

Furthermore, to explore the potential mechanism of PDL against influenza A virus in vivo, mice were intranasally inoculated with $5 \mathrm{LD}_{50}$ of influenza $A$ virus and were administrated by gavage with PDL (5.5 or $4 \mathrm{ml} / \mathrm{kg} /$ day), ribavirin ( $80 \mathrm{mg} / \mathrm{kg} /$ day), or saline daily for 6 days. After treatment for 6 days, all mice were sacrificed and the lung tissues were harvested. The lung index (the ratio of the lung weight to the body weight) was recorded in each mouse. Every lung tissue of each mice was divided into two parts: one for the hematoxylin and eosin (H\&E) staining, and the other for analyzing the expression of inflammatory cytokines by RT-qPCR. H\&E staining was performed as previous described [19]. The histopathological scores of the lung were evaluated as previously described [20].

RT-qPCR analysis of the expression of inflammatory cytokines

Total RNA was extracted using RNA extraction reagent (R401) (Vazyme, China) according to the manufacturer's instructions. cDNA synthesis was performed with HiScript II Q RT Supermix (R222) (Vazyme, China) according to the manufacturer's instructions. qPCR was conducted with AceQ Universal SYBR qPCR Master Mix (Vazyme, China). qPCR was performed as follows: $5 \mathrm{~min}$ at $95^{\circ} \mathrm{C}$, followed by 42 cycles of $10 \mathrm{~s}$ at $95^{\circ} \mathrm{C}$ and $60 \mathrm{~s}$ at $60^{\circ} \mathrm{C}$, and a melting curve step. Primers were synthesized by Genscript (Nanjing, China) and are detailed in Table 1. Relative transcript quantities were calculated using the $2^{-\triangle \triangle C t}$ method with GAPDH as a reference. The primer sequences for QPCR were obtained as required.

Western blot analysis

Western blot assay was performed as described previously [17]. Briefly, A549 cells were infected with influenza A virus and then were exposed to PDL or RBV for $24 \mathrm{~h}$. After treatment, cells were lysed with RIPA lysis buffer (Beyotime, China) to get total protein lysates. Protein samples were separated by $10 \%$ or $12 \%$ SDS-PAGE gels, and then transferred onto nitrocellulose (NC) membrane ( $0.45 \mu \mathrm{m}$, Millipore) using a tank transfer system (Bio-Rad). The membranes were blocked for $1 \mathrm{~h}$ in $5 \%$ BSA in Tris-buffered saline (20 mM Tris, $166 \mathrm{mM} \mathrm{NaCl}$, and $0.05 \%$ Tween 20, $\mathrm{pH}$ 7.5) and then incubated with primary antibodies overnight at $4^{\circ} \mathrm{C}$, and finally incubated with a horseradish peroxidase-conjugated species-specific secondary antibodies at room temperature for $1 \mathrm{~h}$. Bands were visualized using an enhanced chemiluminescence kit (Millipore) with a Molecular Imager SH-523 System (ShenHua, HangZhou). Quantification relative to ACTB by densitometric analysis was performed using Quality One software (BioRad).

\section{Statistical analysis}

All data are expressed as the mean \pm standard deviation (S.D.). For multiple groups, statistical difference was evaluated by one-way analysis of variance (ANOVA) or Student's t-test. Differences in the survival 
rate between groups were analyzed using the Log-rank test. $P$-values of less than or equal to 0.05 were considered statistically significant.

\section{Results}

HPLC profile of PDL

To confirm whether PDL contains the main bioactive ingredients from four Chinese herbs (Taraxacum mongolicum Hand.-Mazz., Scutellaria baicalensis Georgi, Corydalis bungeana Turcz. and Radix Isatidis seu Baphicacanthii), UPLC-MS analysis was performed as previous described [13]. Compared with the mixed standard materials, the nine effective constituents of PDL including (a) adenosine, (b) (r,s)epigoitrin, (c), chlorogenic acid, (d) caffeic acid, (e) cichoric acid, (f) scutellarin, (g) baicalin, (h) oroxylin A, (i) wogonoside were identified and quantified (Fig. 1 and 2). The concentrations of these bioactive components in PDL were $0.3074 \mathrm{mg} / \mathrm{ml}, 0.0830 \mathrm{mg} / \mathrm{ml}, 0.0341 \mathrm{mg} / \mathrm{ml}, 0.0995 \mathrm{mg} / \mathrm{ml}, 0.3606 \mathrm{mg} / \mathrm{ml}$, $0.0786 \mathrm{mg} / \mathrm{ml}, 7.7628 \mathrm{mg} / \mathrm{ml}, 0.3402 \mathrm{mg} / \mathrm{ml}$ and $0.3173 \mathrm{mg} / \mathrm{ml}$, respectively. This result is consistent with the reported literatures $[13,15]$, suggesting that the stability and repeatability of ingredients in PDL.

In vitro anti-influenza viral effect of PDL

All antiviral efficacy tests of PDL are conducted under non-toxic dose conditions ( $\mathrm{TC}_{50}$ of PDL was 7.94 $\mu \mathrm{l} / \mathrm{ml}$ ). PDL significantly inhibit the replication of influenza H1N1 (FM1, PR8, WSN), and H3N2 viruses and showed a good protective effect on cell pathogenicity caused by influenza $A$ virus. The $\mathrm{IC}_{50}$ of PDL against FM1, PR8, WSN, and H3N2 is $0.55 \mu \mathrm{l} / \mathrm{ml}, 1.10 \mu \mathrm{l} / \mathrm{ml}, 0.98 \mu \mathrm{l} / \mathrm{ml}$, and $1.88 \mu \mathrm{l} / \mathrm{ml}$, respectively. The selectively index (SI) was $14.4,7.22,8.10$ and 4.22 , respectively. Ribavirin $(50 \mu \mathrm{g} / \mathrm{ml}, 10 \mu \mathrm{g} / \mathrm{ml}$ and 5 $\mu \mathrm{g} / \mathrm{ml})$ and oseltamivir $(8 \mu \mathrm{g} / \mathrm{ml}, 4 \mu \mathrm{g} / \mathrm{ml}$ and $2 \mu \mathrm{g} / \mathrm{ml})$ could completely inhibit the replication of the above influenza virus.

In vivo anti-influenza viral effect of PDL

To determine the optimal dose of PDL, the effect of PDL on the growth of mice body weight was analyzed. As shown in Fig. 3A, compared with the control group, $8.0 \mathrm{ml} / \mathrm{kg}$ of PDL $(\mathrm{H})$ had obvious effects on the increase of mice body weight, $6.0 \mathrm{ml} / \mathrm{kg}$ of PDL (M) showed a slight effect on the growth of body weight of mice, while $4.0 \mathrm{ml} / \mathrm{kg}$ of PDL $(\mathrm{L})$ has no obvious effect. However, generally, the effect of PDL (4.0-8.0 ml/ kg) on the growth of body weight of mice was markedly less than that the positive drug ribavirin, which has a significant effect on mice body weight [3]. Furthermore, we further investigated the effect of PDL against influenza A virus in vivo according to these above dosages. To evaluate the effect of PDL against influenza A virus in vivo, the clinical signs, food intake, and weight change of mice treated with different drugs or model were recorded daily. During the observation period, compared with the virus-infected group, the clinical symptoms of the mice-treated with PDL $(8.0$ and $6.0 \mathrm{ml} / \mathrm{kg}$ ) were significantly improved and the onset time was also markedly delayed. After 3 days post-infection, the food intake of the mice-infected group was significantly decreased, while the treatment with PDL or RBV could significantly prevent the rapid decline in the diet of mice (Fig. 3B). Moreover, compared with the 
virus-infected group, PDL treatment could dose-dependently delay the weight loss of mice. As shown in Fig. 3C, 4 day post-infection, the weight body of the virus-infected group or PDL $(4.0 \mathrm{ml} / \mathrm{kg}$ ) began to gradually decrease compared to initial weight, while the body weight of the mice treated with PDL (6.0 $\mathrm{ml} / / \mathrm{kg}$ and $8.0 \mathrm{ml} / \mathrm{kg}$ ) delay to decline until the 6th day post-infection. Positive drug RBV could significantly prevent the weight loss of mice. In addition, we also found that all mice infected with H1N1 virus died at between 8- and 11-days post-infection with $10.1 \pm 2.2$ days of median survival time (Table 1). However, PDL could dose-dependently promote the survival of the mice-infected with virus and prolonged the survival time (Table 1). Notably, although RBV treatment couldn't prevent the weight loss caused by lethal H1N1 virus, but could provide $100 \%$ protection for lethal H1N1 virus (Table 1).

PDL effectively improves acute lung injury caused by influenza A virus

To further confirm the antiviral effect of PDL on influenza A H1N1 virus in vivo, the lung edema was evaluated by the lung index at 8 days post-infection. As shown in Fig. 4, the lung index of the virusinfected group was markedly increased, which was significantly higher than that of the control group. However, PDL treatment could significantly decrease the lung index, suggesting the protective effect of PDL on acute lung injury caused by influenza A H1N1 virus. Moreover, the inhibition effect of PDL (0.55 $\mathrm{ml} / \mathrm{kg}$ ) on the lung index is equivalent to the positive drug RBV, but significantly stronger than the SHL treatment group. H\&E staining also indicated that PDL treatment could effectively improve the pathological changes of lung tissue caused by influenza A virus. As shown in Fig. 4, influenza A H1N1 virus could cause severe lung injury with a diffuse swelling, severe cell necrosis, alveolar cavity collapse, alveolar thickening, severe infiltration of large inflammatory cells, and hemorrhage in the model group, while PDL treatment could reduce the above pathological changes, which was also been confirmed by pathology scores. These results indicated that PDL treatment showed significant protective effects on acute lung injury caused by influenza A virus.

The in vivo inhibitory effect of PDL on the inflammatory cytokines

It has been demonstrated that the pro-inflammatory cytokines play a crucial role in the acute lung injury and ARDS caused by severe influenza A virus [21, 22]. Therefore, we examined the expression levels of the proinflammatory factors in lung tissue. As shown in Fig. 5, compared with the virus-infected group, PDL and RBV treatment could markedly inhibit the transcription of TNF-a, IP10, IL-10, and IFN- $\gamma$ mRNA, but the inhibitory effect of PDL treatment on these above cytokines is stronger than that of RBV treatment. Notably, compared with the virus-infected group, PDL and RBV treatment also significantly increased the expression of IL-6, but the expression levels of IL-6 in the PDL treatment group was slightly weaker than that in the RBV treatment group. Taken together, these above results indicated that PDL treatment can significantly inhibit the expression of inflammatory cytokines caused by influenza virus.

The potential mechanism of PDL against acute lung injury caused by influenza A virus

Toll-like receptors (TLRs) as the host's first line of defense against pathogenic microbial infections play a crucial role in clearing virus and inhibiting the excessive inflammatory responses caused by virus[23, 24]. 
In addition, it has been widely reported that TLR3-mediated signaling pathway are involved in regulating host inflammatory responses to influenza A virus [22-26]. To further investigate the underlying antinflammatory mechanisms of PDL, the expression levels of TLR3, MyD88, IRF7, and TRAF3 mRNA in lung tissue were analyzed. As shown in Fig. 6, compared with the virus-infected group, the mRNA levels of TLR3, MyD88, IRF7, and TRAF3 in the lung tissue were significantly decreased in the PDL-treated group. Notably, the pharmacological effect of PDL on these above inflammatory signaling pathways is almost similar to that of the positive antiviral drug, RBV. To further confirm the above effect of PDL, A549 human lung cancer cells were selected to investigate the anti-inflammatory mechanism of PDL by immunoblotting. As shown in Fig. 7, influenza A virus infection could markedly increase the expression of TLR3, TLR7, MyD88, IRF7, TRAF3 and TRAF6, while PDL treatment could significantly inhibit the expression of these above proteins. Furthermore, the results of viral NP gene expression also indicated that PDL can effectively inhibit the replication of influenza virus as RBV, further confirming the antiviral effect of PDL. These results suggested that PDL may exert the antiviral or anti-inflammatory effect by suppressing TLR3/MyD88/NF-KB signaling pathway.

\section{Discussion}

Influenza A virus as one common respiratory pathogen could cause global pandemics and epidemics in the world [27-28]. Due to the high mortality and morbidity of influenza A virus infection, influenza is still a major public health problem. Although preventive administration with antiviral drugs such as oseltamivir, zanamivir, amantadine could effectively improve outcomes in patients infected by influenza viruses, there are no effective strategies to treat severe seasonal influenza and highly pathogenic avian influenza $[8,29]$. It has been demonstrated that the "cytokine storm" and excessive inflammatory response caused by lethal influenza virus are responsible for its high mortality and morbidity $[21,22,30$, 31]. Therefore, inhibiting the inflammatory response caused by influenza A virus may become an effective therapy for treatment of this diseases $[8,29]$. Traditional Chinese herbal medicine (TCM) such as honeysuckle, forsythia, skullcap and dandelion were widely used in clinical treatment of infectious disease for long time. In addition, Chinese patent medicine, such as Yin-Qiao-San, Gegen-qianliandecotion, and Ching-fang-pai-tu-san, which is composed of traditional Chinese herbal medicine, has been widely used to treat common colds, influenza, and upper respiratory tract infection [20,32-34]. PDL as a well-known Chinese patent medicine, which is composed of Taraxacum mongolicum Hand.-Mazz, Scutellaria baicalensis Georgi, Corydalis bungeana Turcz, and Radix Isatidis, has been widely used to treat pharyngitis, tonsillitis and upper respiratory tract infection in clinical due to its remarkable antiinflammatory effect[13-15]. Sometimes, it is clinically used for the treatment of pandemic colds. However, whether it has an anti-influenza virus effect has not been reported. In this present study, our results for the first time demonstrated that PDL could effectively inhibit the replication of seasonal severe influenza A virus including H1N1 and H3N2 in vitro. Furthermore, our results also indicated that PDL exhibited a good anti-influenza activity in vivo, which has been confirmed by reducing the mortality and extending the survival time. Moreover, PDL treatment could significantly alleviate the pulmonary edema and pathological scores caused by influenza A virus and markedly prevent the weight loss. In addition, 
mechanistic study indicated that the anti-influenza effect of PDL in vivo might be highly associated with the inhibition of the expression of inflammatory cytokines such as TNF-a, IL-6, IP-10, IFN- $\gamma$, and IL-1 $\beta$ via TLR3-MyD88-IRAK4-TRAF6 signaling pathway.

An emerging of studies has reported that excessive inflammatory response is the main reason for the high clinical mortality of influenza A virus infection. For example, high levels of proinflammatory cytokines such as TNF-a, IL-6, and IP-10 in sera of patients infected with severe seasonal influenza or highly pathogenic avian influenza viruses were commonly found [11, 30-31]. Therefore, inhibiting the excessive inflammatory responses may effectively improve the prognosis of influenza A virus infection. Recently, it has been demonstrated that PDL could effectively suppress the expression of proinflammatory factors TNF- $a$ and IL-6 in LPS-induced respiratory injury model through inhibiting TLR4 signaling pathway or regulating the disturbed metabolic pathway [14]. Similar to the results reported in the above literature, we also found that PDL treatment significantly inhibit the expression of proinflammatory cytokines including TNF-a, IP-10, IFN- $y$, and IL-10 in the mouse model of influenza A virus infection. However, contrary to the inhibitory effect of PDL treatment on IL-6 expression observed in the aforementioned LPS-induced acute injury model in mice, our results showed that PDL treatment could significantly upregulate IL- 6 expression. It has been demonstrated that IL- 6 as a well-known cytokine produced by many different cells has a variety of biological functions, including antiviral, promoting acute phase proteins expression, stimulating B cell differentiation to producing antibody, activating human T cells, and enhancing NK cell activity. Compared with the virus-infected group or other positive control, the antibody titers of PDL treatment against $\mathrm{H} 1 \mathrm{~N} 1$ virus was the highest, while the virus titers in lung tissue was the lowest, suggesting that high levels of IL-6 in PDL treatment group might be associated with the high levels of antibody against influenza A virus. However, the underlying mechanism of PDL treatment how to induce IL- 6 expression and enhance the antibody titer against influenza A virus remains to be further investigated. The delineation of the relationship of IL- 6 and antibody titers will help to understand the potential mechanism of PDL against influenza A virus.

Toll-like receptors (TLRs) as key pattern recognition receptors for recognizing virus play a critical role in triggering host antiviral responses. In mammals, TLR3, 7, 8 and 9 are widely involved in recognizing viral nucleic acids [22-24]. Among them, TLR3 as a major receptor for recognizing viral double-stranded RNA (dsRNA) plays a crucial role in protecting the host against viruses [25]. However, it has also been observed that the activation of TLR3 signaling might be deleterious in some viral infections. For example, it has been reported that TLR3-deficient mice survived influenza A virus infection better than wild-type mice due to lower levels of inflammatory mediators, suggesting that TLR3 plays a detrimental role of TLR3-mediated inflammatory responses in influenza virus-induced acute pneumonia[35]. Recently, Huo et al also demonstrated that lethal influenza A virus preferentially activates TLR3 and triggers severe inflammatory responses [22]. Furthermore, the effect of TLR3 promoting acute lung injury in the development of ARDS-like pathology has also been demonstrated using TLR3-gene deficient mice which exhibited less acute lung injury, activation of apoptotic cascades and reduced neutrophil influx. In view of this major information, we first investigated the effect of PDL treatment on the expression of TLR receptors. Notably, compared with the virus-infected mice, PDL treatment significantly inhibited the 
expression of TLR3 mRNA, but markedly promoted the transcription of TLR4 and TLR7 mRNA, suggesting that PDL has different effects on TLRs expression. Consistent with the effect of PDL on TLR3, PDL treatment markedly inhibited the expression of MyD88, IRAK4, and TRAF3 mRNA. In addition, we found that PDL treatment significantly upregulated the expression of TRAF6 mRNA. Due to TLR3 signaling pathway is MyD88-independent pathway, the down-regulation of MyD88 caused by PDL treatment suggested that PDL might exert its pharmacological effect by other MyD88-dependent signaling pathway. These above results indicated that the anti-influenza effect of FFYH against viral pneumonia may be achieved through regulating the TLR7/MyD88 signaling pathway.

\section{Conclusion}

In summary, our results showed that PDL not only can effectively inhibit the replication of influenza $\mathrm{A}$ virus, including $\mathrm{H} 1 \mathrm{~N} 1, \mathrm{H} 3 \mathrm{~N} 2, \mathrm{H} 5 \mathrm{~N} 1, \mathrm{H} 7 \mathrm{~N} 9$, and $\mathrm{H} 9 \mathrm{~N} 2$ in vitro, but also markedly improve the survival rate of mice caused by lethal influenza virus. Mechanistic researches indicated that PDL might exert its potential therapeutic effect on acute lung injury caused by influenza A virus through regulating the TLR3 independent of MyD88 signaling pathway to inhibiting of excessive inflammatory response (Fig. 7). These findings suggested that PDL might be effective for treatment of influenza A virus.

\section{Abbreviations}

PDLIPudilan xiaoyan oral liquid; TLR-30Toll-like receptor 3;TLR-70Toll-like receptor 7; TLR-8DToll-like receptor 8; TLR-9Toll-like receptor 9;MyD88®Myeloid differentiation primary response gene (88); IRF7] Interferon regulator factor 7; MDCK \Madin-Darby canine kindey; TNF-a冈Tumor necrosis factor-a; IP10

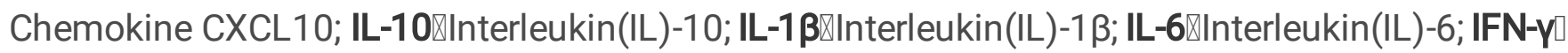
Interferon-ү; RT-qPCR $₫$ Quantitative reverse transcription PCR; IRAK4『Interleukin-1 receptor-associated kinase 4; RT-PCR $\searrow$ Reverse transcription PCR; TRAF3 $₫$ TNF receptor-associated factor (TRAF) 3; NF-KB

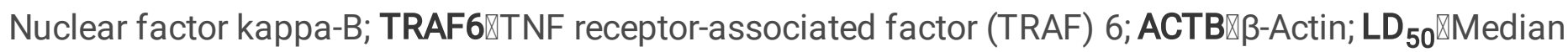
lethal dose; BSL-2 or BSL-3®Biosafety laboratory-2 or -3; $\mathbf{T C}_{\mathbf{0}} \otimes$ No toxic concentration; $\mathbf{T C}_{\mathbf{5 0}} \otimes$ Median toxic concentration; NC $₫$ Nitrocellulose membrane; HPLC $₫$ High Performance Liquid Chromatography; RBV Ribavirin; AIV\Influenza A virus.

\section{Declarations}

\section{Ethics approval and consent to participate}

All animal experiments were approved by the Ethics Committee of Yangzhou University (202010002) according to the Chinese Animal Protection Act and the National Research Council Criteria.

\section{Consent for publish}

All authors have agreed to publish it in the journal of Chinese Medicine. 
Availability of data and materials

All data are available from the corresponding author upon request.

\section{Competing interests}

All authors declare no conflict of interest.

\section{Funding}

This study was supported by the National Key New Drug Creation and Manufacturing Program, Ministry of Science and Technology (2019ZX09731001); the Program for New Century Excellent Talents in University (Program No. NCET-12-0975), 2020 Yangzhou University undergraduate Research and Innovation Program (202111117111Y).

\section{Author contributions}

Pinghu Zhang designed the study, analyzed the data, and wrote the manuscript. Zhihui Zheng and Yuqian Zhang performed the experiments and collected the data. Gang Tian performed HPLC analysis for PDL. Xiaoquan Wang identified the virus strains and determined the virus titers. Li Ming, Wei Hailin, Wang Wenlei, Su qin, Wang Zehua, and Min Rui conducted the animal experiments and collected the data.

\section{Acknowledgements}

We thank Mr. Mohammed Salem for improvement language and Dr. Kaituo Liu, Jijun Gan, Xiaoyan Tian in the College of Veterinary Medicine \& Jiangsu Provincial Key Laboratory of Human Zoonosis for assistance in animal experiments.

\section{References}

1. Short, K.R., et al., Pathogenesis of influenza-induced acute respiratory distress syndrome. The Lancet Infectious Diseases, 2014. 14(1): 57-69.

2. Fukuyama, S. and Y. Kawaoka, The pathogenesis of influenza virus infections: the contributions of virus and host factors. Current Opinion in Immunology, 2011. 23(4): 481-486.

3. Peiris, J.S.M., et al., Innate immune responses to influenza A H5N1: friend or foe? Trends in Immunology, 2009. 30(12): 574-584.

4. Watanabe, T., et al., Pandemic potential of avian influenza A (H7N9) viruses. Trends in Microbiology, 2014. 22(11): 623-631.

5. Park, J.-E. and Y. Ryu, Transmissibility and severity of influenza virus by subtype. Infection, Genetics and Evolution, 2018. 65: 288-292. 
6. Lobo, S.M., et al., Excess mortality is associated with influenza A (H1N1) in patients with severe acute respiratory illness. Journal of Clinical Virology, 2019. 116: 62-68.

7. Damjanovic, D., et al., Immunopathology in influenza virus infection: Uncoupling the friend from foe. Clinical Immunology, 2012. 144(1): 57-69.

8. Dunning, J., et al., Antiviral combinations for severe influenza. The Lancet Infectious Diseases, 2014. 14(12): 1259-1270.

9. Tian, L., et al., Evaluation of the anti-neuraminidase activity of the traditional Chinese medicines and determination of the anti-influenza A virus effects of the neuraminidase inhibitory TCMs in vitro and in vivo. J Ethnopharmacol, 2011. 137(1): 534-42.

10. To, K.K.W., et al., The emergence of influenza A H7N9 in human beings 16 years after influenza A H5N1: a tale of two cities. The Lancet Infectious Diseases, 2013. 13(9): 809-821.

11. Yang, Y., et al., Epidemiological and clinical characteristics of humans with avian influenza A (H7N9) infection in Guangdong, China, 2013-2017. International Journal of Infectious Diseases, 2017. 65: 148-155.

12. Abdelwhab, E.M., J. Veits, and T.C. Mettenleiter, Biological fitness and natural selection of amantadine resistant variants of avian influenza H5N1 viruses. Virus Research, 2017. 228: 109-113.

13. Feng, L., et al., Pudilan xiaoyan oral liquid alleviates LPS-induced respiratory injury through decreasing nitroxidative stress and blocking TLR4 activation along with NF-KappaB phosphorylation in mice. J Ethnopharmacol, 2018. 214: 292-300.

14. Cheng, L., et al., Evaluation of the effect of a toothpaste containing Pudilan extract on inhibiting plaques and reducing chronic gingivitis: A randomized, double-blinded, parallel controlled clinical trial. J Ethnopharmacol, 2019. 240: 111870.

15. Tian, G., et al., GC-MS based metabolomic profiling of lung tissue couple with network pharmacology revealed the possible protection mechanism of Pudilan Xiaoyan Oral Liquid in LPSinduced lung injury of mice. Biomed Pharmacother, 2020. 124: 109833.

16. Thakur A.K. and Fezio W.L. A computer program for estimating LD50 and its confidence limits using modified Behrens-Reed-Muench cumulant method. Drug Chem Toxicol. 1981, 4(3):297-305.

17. Zhang et al., w09, a novel autophagy enhancer, induces autophagy-dependent cell apoptosis via activation of the EGFR-mediated RAS-RAF1-MAP2K-MAPK1/3 pathway. Autophagy, 2017, 13(7): 10931112.

18. Yan, H., et al., Cirsimaritin inhibits influenza A virus replication by downregulating the NF-kB signal transduction pathway. Virol J, 2018. 15, 88. https://doi.org/10.1186/s12985-018-0995-6. 
19. Zhang et al, Evaluation of mitochondrial toxicity in Marmota himalayana treated with metacavir, a novel 2',3'-dideoxyguanosine prodrug for treatment of hepatitis B Virus, Antimicrob Agents Chemother. 2011, 55(5):1930-1936.

20. Rong, R., et al., Mahuang-Xixin-Fuzi decoction reduces the infection of influenza A virus in KidneyYang deficiency syndrome mice. J Ethnopharmacol, 2016. 192: 217-224.

21. Lobo, S.M., et al., Excess mortality is associated with influenza A (H1N1) in patients with severe acute respiratory illness. J Clin Virol, 2019. 116: 62-68.

22. Huo, C., et al., Lethal influenza A virus preferentially activates TLR3 and triggers a severe inflammatory response. Virus Res, 2018. 257: 102-112.

23. Elshabrawy, H.A., et al., TLRs, future potential therapeutic targets for RA. Autoimmun Rev, 2017. 16(2): 103-113.

24. Arora, S., et al., TLRs in pulmonary diseases. Life Sciences, 2019. 233: 116671.

25. Karpala, A.J., J.W. Lowenthal, and A.G. Bean, Activation of the TLR3 pathway regulates IFNbeta production in chickens. Dev Comp Immunol, 2008. 32(4): 435-44.

26. Zhang, S.Y., et al., TLR3 immunity to infection in mice and humans. Curr Opin Immunol, 2013. 25(1): 19-33.

27. Ludwig, S., et al., Influenza, a One Health paradigm--novel therapeutic strategies to fight a zoonotic pathogen with pandemic potential. Int J Med Microbiol, 2014. 304(7): 894-901.

28. Park, J.E. and Y. Ryu, Transmissibility and severity of influenza virus by subtype. Infect Genet Evol, 2018. 65: 288-292.

29. Ison, M.G., Antivirals and resistance: influenza virus. Current Opinion in Virology, 2011. 1(6): 563573.

30. Svitek, N., et al., Severe seasonal influenza in ferrets correlates with reduced interferon and increased IL-6 induction. Virology, 2008. 376(1): 53-59.

31. Fukuyama, S. and Y. Kawaoka, The pathogenesis of influenza virus infections: the contributions of virus and host factors. Curr Opin Immunol, 2011. 23(4): 481-486.

32. Hsieh, C.F., et al., Ching-fang-pai-tu-san inhibits the release of influenza virus. J Ethnopharmacol, 2012. 144(3): 533-544.

33. Law, A.H.-Y., et al., Antiviral effect of forsythoside A from Forsythia suspensa (Thunb.) Vahl fruit against influenza A virus through reduction of viral M1 protein. J Ethnopharmacol, 2017. 209: 236-247. 
34. Ma, Q., et al., Liu Shen Wan inhibits influenza a virus and excessive virus-induced inflammatory response via suppression of TLR4/NF-KB signaling pathway in vitro and in vivo. J Ethnopharmacol, 2020. 252: 112584.

35. Le Goffic R, Balloy V, Lagranderie M, et al. Detrimental contribution of the Toll-like receptor (TLR)3 to influenza A virus-induced acute pneumonia. PLoS Pathog. 2006, 2(6):e53. doi:

10.1371/journal.ppat.0020053.

\section{Figures}

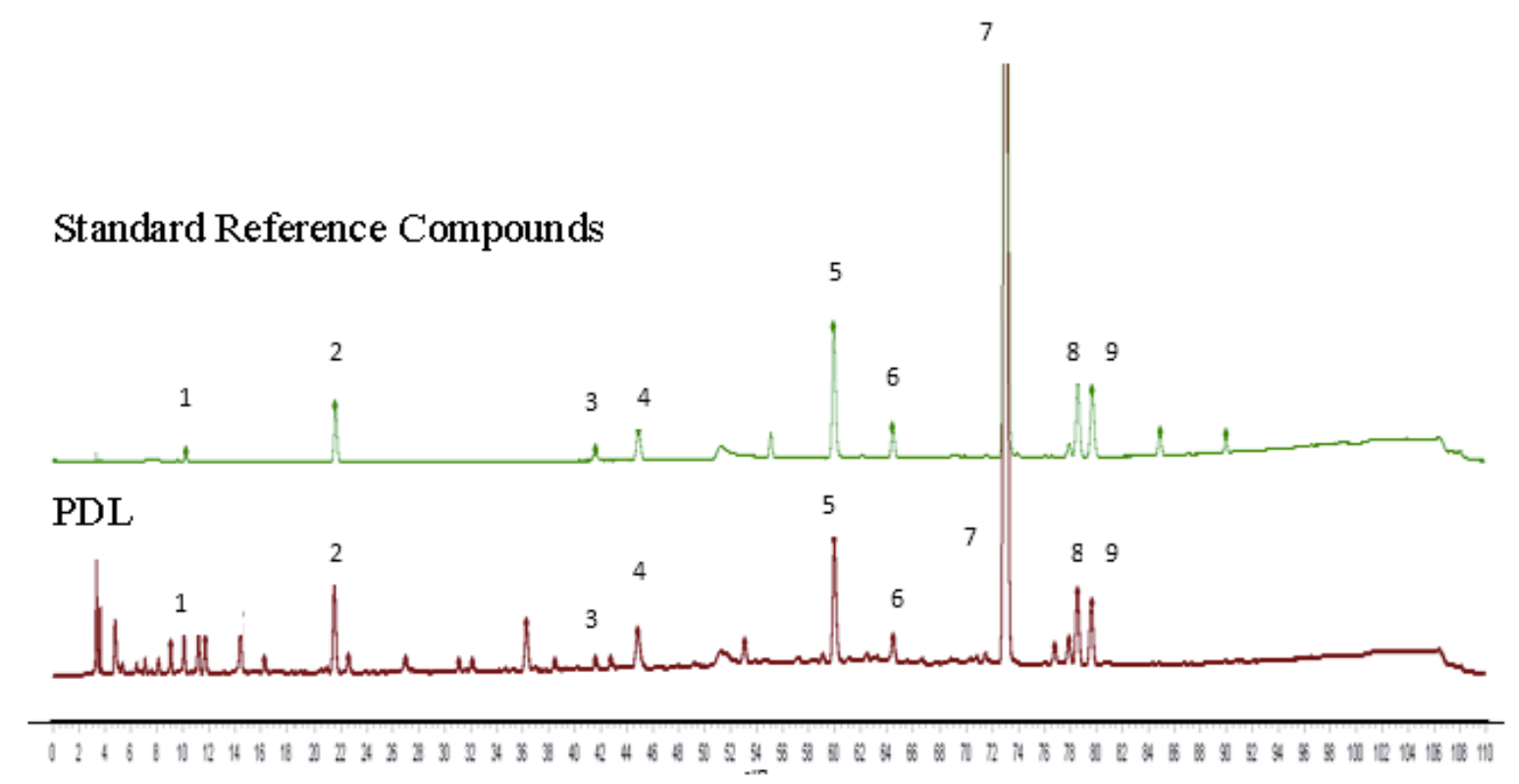

Fig.1 HPLC chromatograms of nine effective constituents in PDL at $230 \mathrm{~nm} .1$. Adenosine, 2. (R,S)-epigoitrin, 3. Chlorogenic acid, 4. Caffeic acid, 5. Cichoric acid, 6. Scutellarin, 7. Baicalin, 8. Oroxylin A, 9. Wogonoside.

\section{Figure 1}

See image above for figure legend 


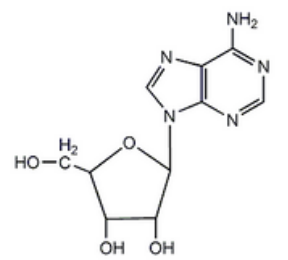

Adenosine

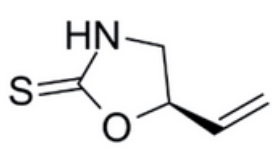

(R,S)-epigoitrin

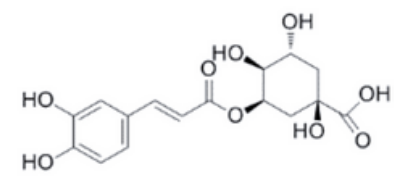

Chlorogenic acid<smiles>O=C(O)C=Cc1ccc(O)c(O)c1</smiles>

Caffeic acid<smiles></smiles>

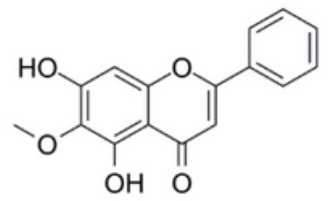

Oroxylin A<smiles>COc1cc(-c2ccccc2)cc(O)c1OC1OC(C(=O)O)C(O)C(O)C1Oc1cccc(O)c1</smiles>

Wogonoside

Fig.2 Chemical structures of nine representative compounds in PDL.

Figure 2

See image above for figure legend 

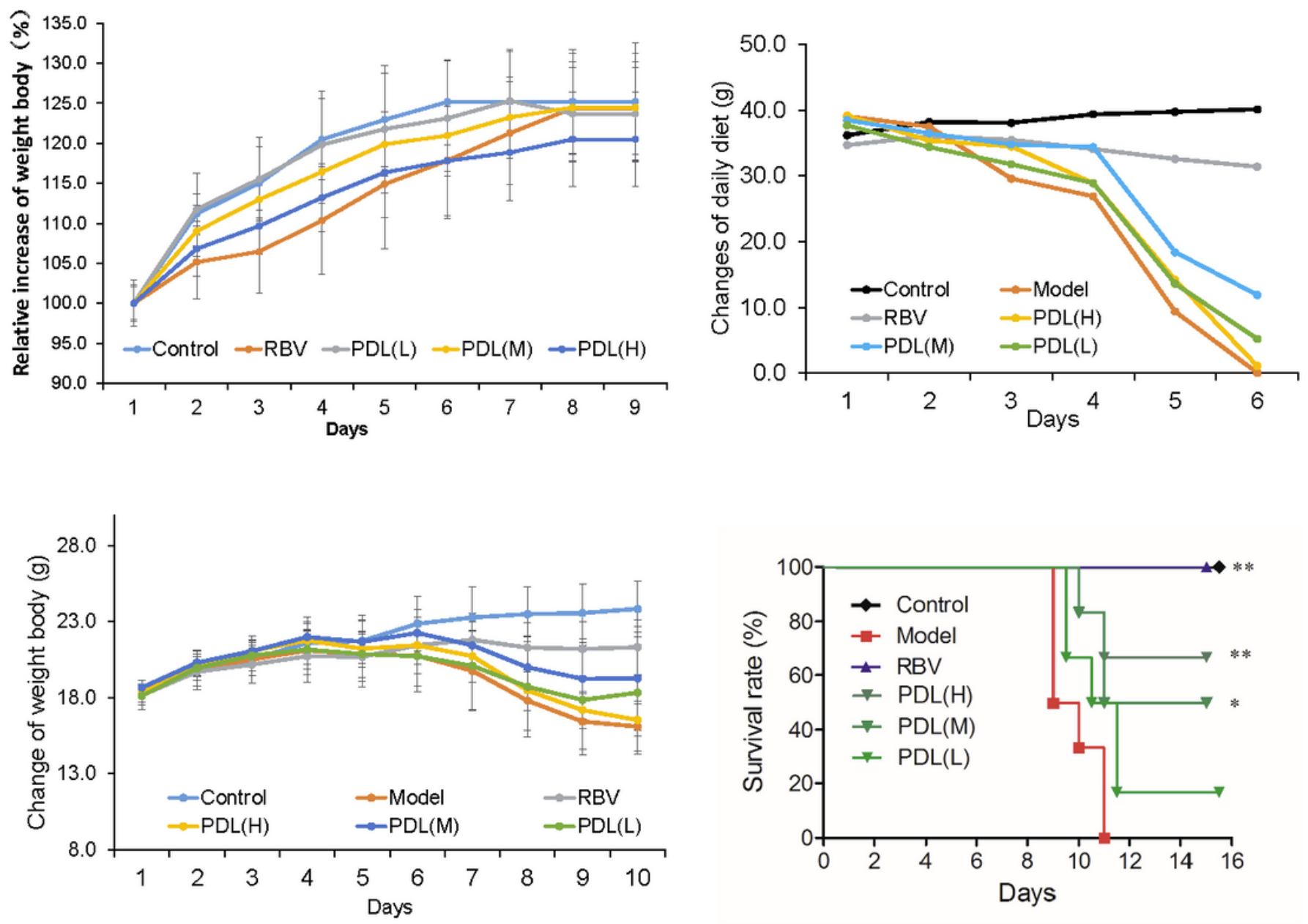

Fig.3 The protective effect of PDL on lethal H1N1 virus in vivo. Mice were inoculated with $5 \mathrm{LD}_{50}$ of virus and then were administrated with orally with PDL $(8.0 \mathrm{ml} / \mathrm{kg}, 6.0 \mathrm{ml} / \mathrm{kg}, 4.0 \mathrm{ml} / \mathrm{kg})$, or ribavirin $(80 \mathrm{mg} / \mathrm{kg})$ for 6 days. All mice were observed daily for 15 days. The weight loss, food intake, and mortality was recorded daily. The protective effects were evaluated by the survival time and the reduction of mortality. ${ }^{*} \mathrm{p}<0.05,{ }^{*} \mathrm{p}<0.01$ vs $\mathrm{H} 1 \mathrm{~N} 1$ virus infection group.

\section{Figure 3}

See image above for figure legend 

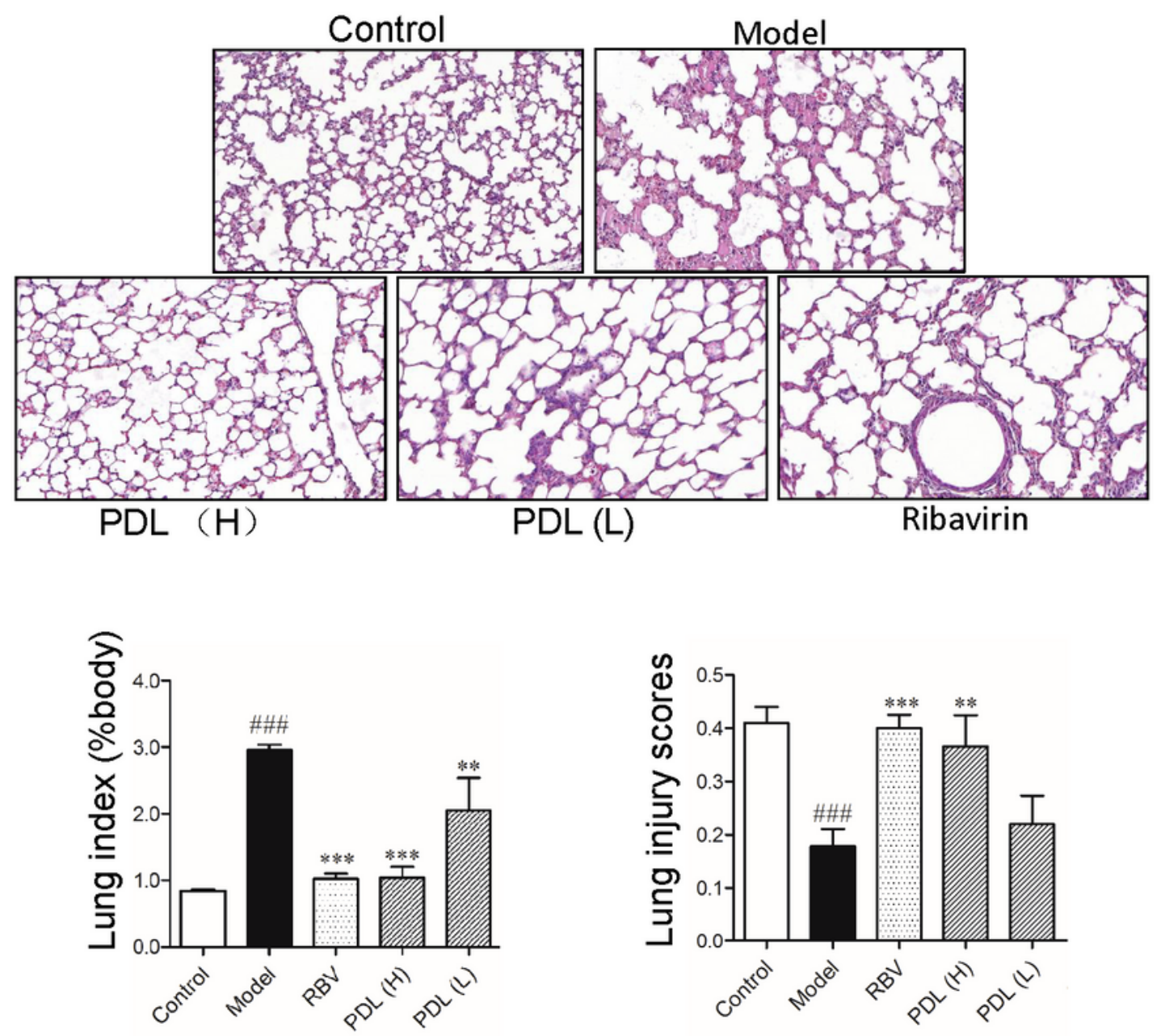

Fig. 4 The in vivo protective effect of PDL on lung injury caused by H1N1 virus infection. Mice were inoculated with influenza virus and then were administrated orally with PDL $(5.5 \mathrm{ml} / \mathrm{kg}$ or $4.0 \mathrm{ml} / \mathrm{kg})$, or ribavirin $(80 \mathrm{mg} / \mathrm{kg})(\mathrm{n}=6)$ for 6 days. And then the mice were sacrificed and the lung tissues were harvested and weighed. The pulmonary edema was evaluated by lung index. Histological changes of lung were analyzed with hematoxylin and eosin staining and photographed $(200 \times)$. ${ }^{*} \mathrm{p}<0.05,{ }^{* * *} \mathrm{p}<0.01$ vs the virus infection group; ${ }^{{ }^{\prime}} \mathrm{p}<0.01$, when compared with the normal group.

\section{Figure 4}

See image above for figure legend 

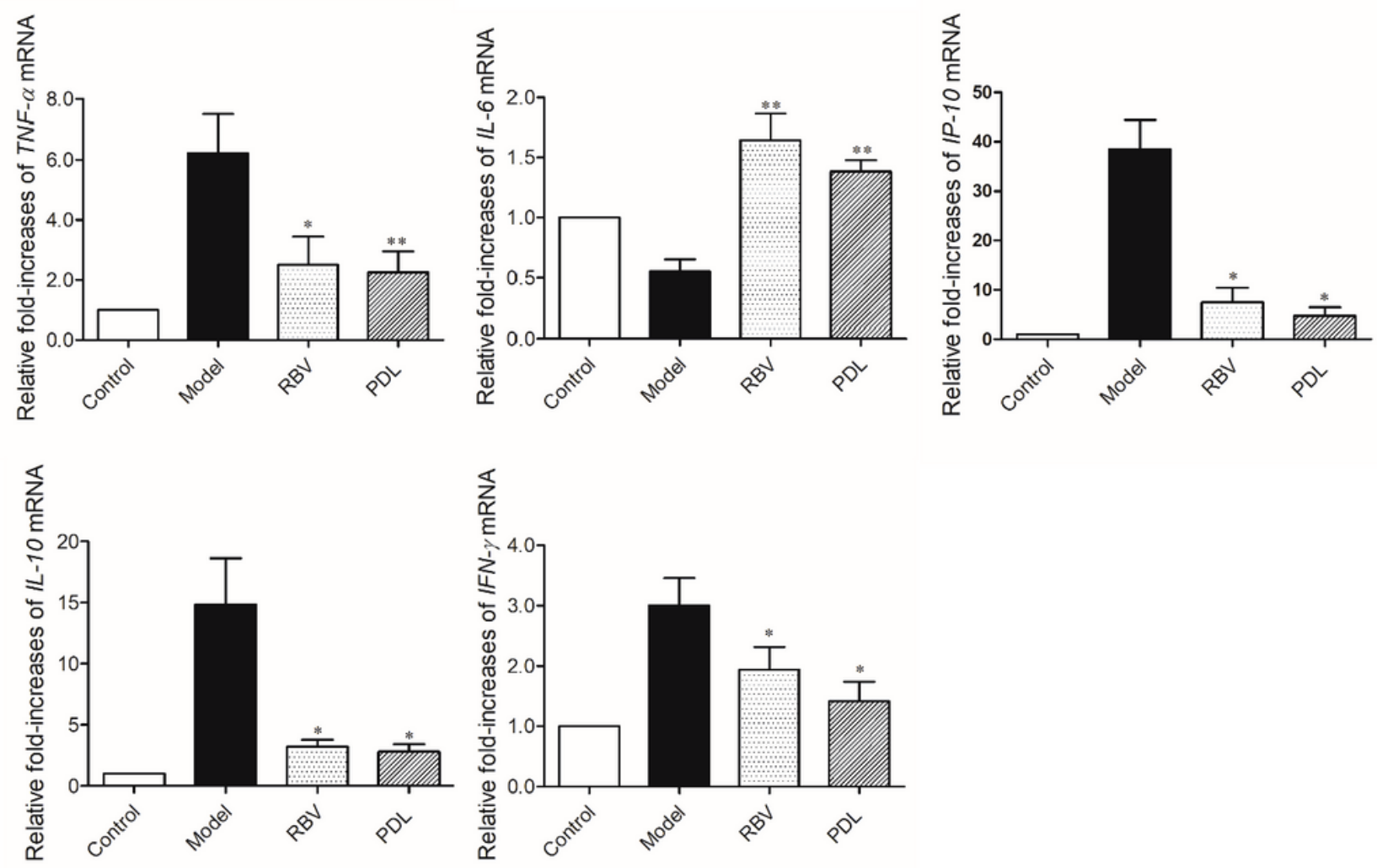

Fig.5 The in vivo inhibitory effect of PDL on the inflammatory cytokines. TNF- $\alpha$, IL-6, IP10, IL-1 $\beta$, IL-10, and IFN- $\gamma$ mRNA caused by lethal influenza virus were quantified by qRT-PCR. Relative fold-increases of these above cytokines were quantified using the $2^{-\Delta \Delta C t}$ method with GAPDH as a reference. ${ }^{*} \mathrm{p}<0.05,{ }^{*} \mathrm{p}<0.01$ vs virus infection.

Figure 5

See image above for figure legend 

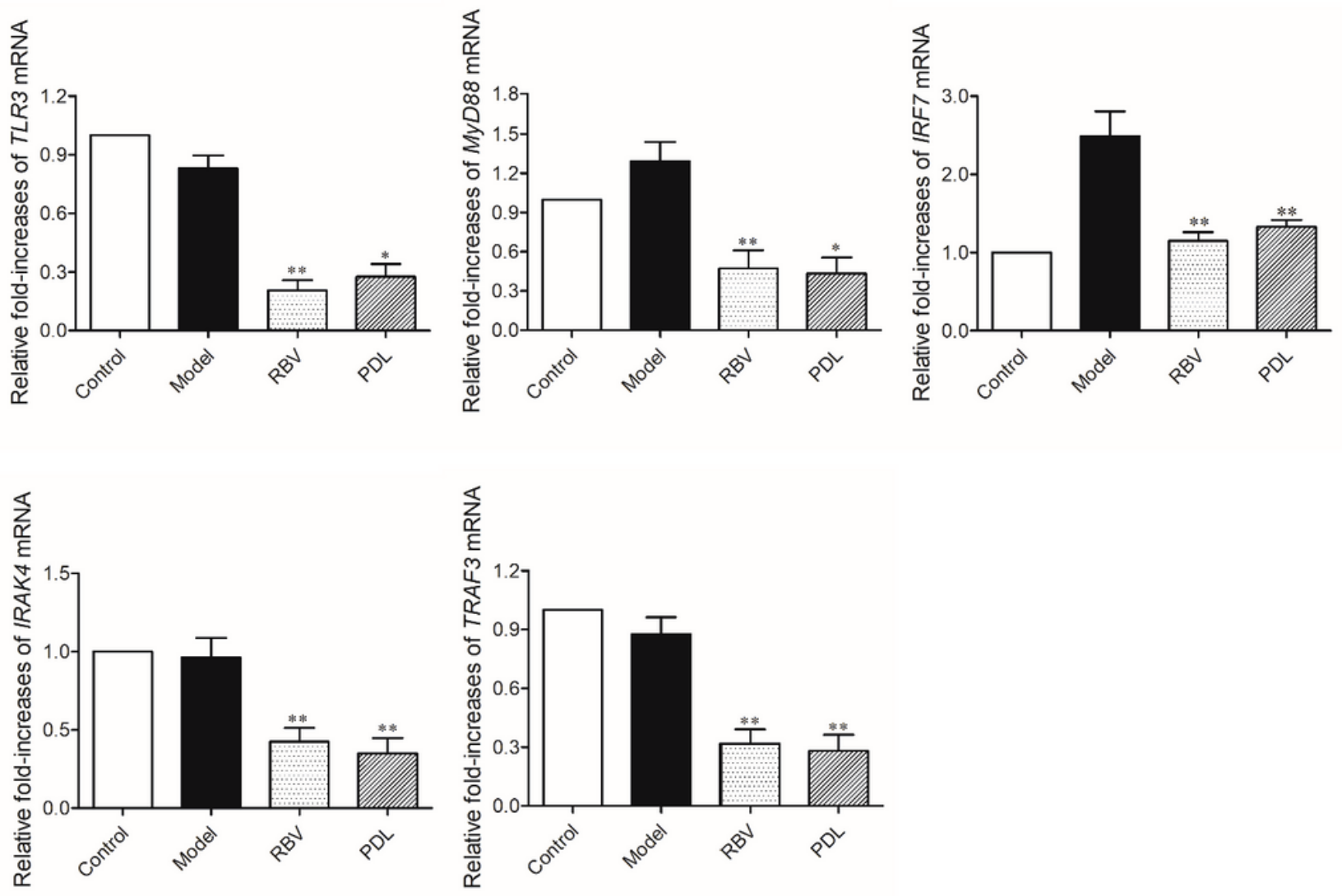

Fig. 6 The potential mechanism of action of PDL against viral pneumonia. Relative transcription levels of inflammation-related signaling pathway, including TLR3, MyD88, IRF7, IRAK4, and TRAF3 mRNA were quantified by qRT-PCR and were analyzed using the $2^{-\Delta \Delta C t}$ method with GAPDH as a reference. ${ }^{*} \mathrm{p}<0.05,{ }^{*} \mathrm{p}<0.01 \mathrm{vs}$ the virus-infected group $(n=6)$.

Figure 6

See image above for figure legend 

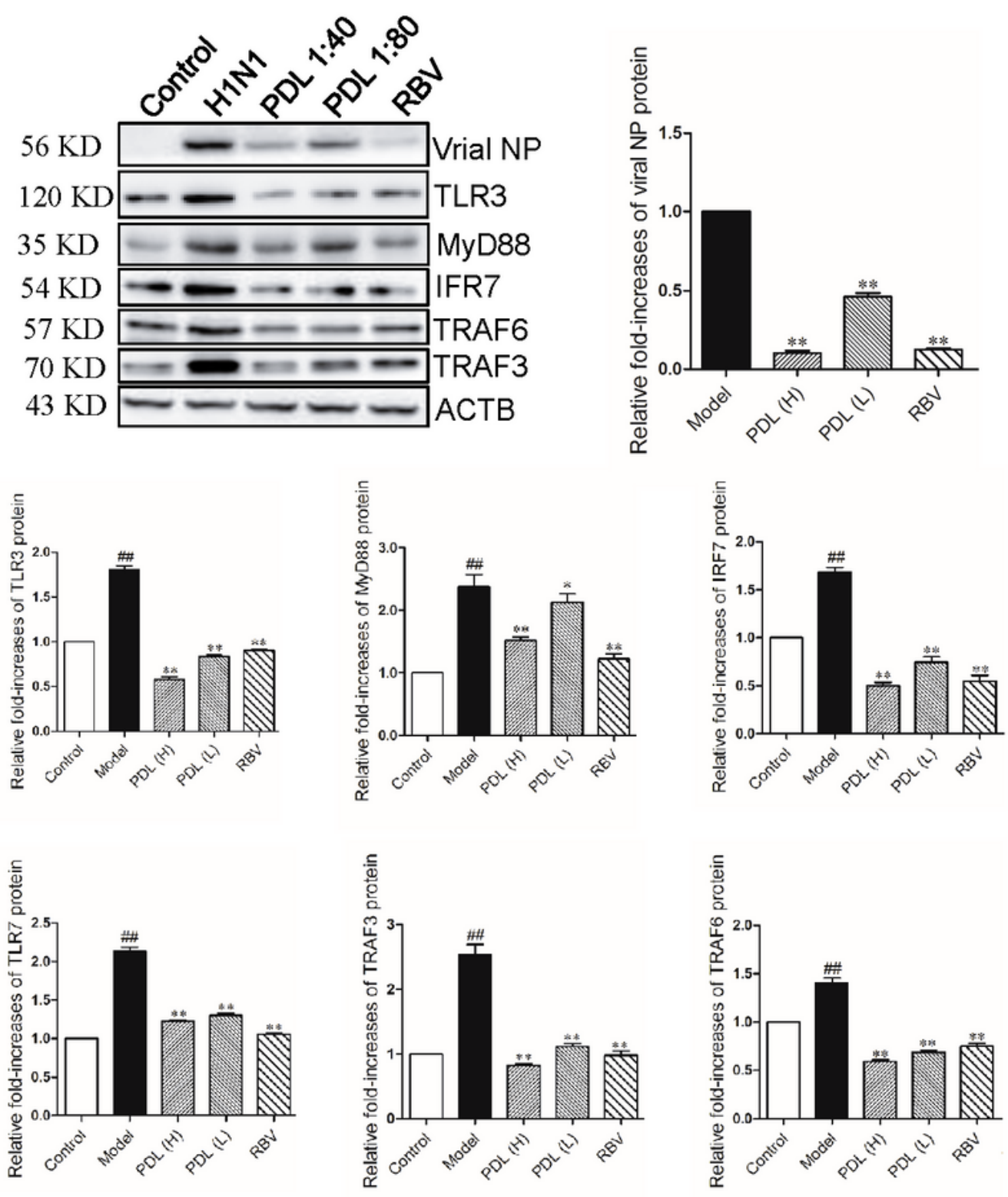

Fig.7 The potential mechanism of PDL against influenza A virus. After infection, A549 cells were treated with PDL or Ribavirin for $24 \mathrm{~h}$, and the expression levels of viral NP, TLR3, MyD88, IRF7, TRAF3 and TRAF6 were examined with immunoblotting. Relative expression levels were quantified with using quality one software (Bio-Rad). ${ }^{\#} \mathrm{p}<0.01$, compared with normal control group; ${ }^{*} \mathrm{p}<0.05$, or ${ }^{* * *}$ $\mathrm{p}<0.01$, compared with the virus-infected group.

\section{Figure 7}

See image above for figure legend 